\title{
Obesity and Weight Regulation
}

\author{
Martin Wabitsch, Daniel Tews, Michaela Keuper, Carsten Posovszky, Christian \\ Denzer, Anja Moss, Julia von Schnurbein and Pamela Fischer-Posovszky \\ Division of Pediatric Endocrinology and Diabetes, Department of Pediatrics and Adolescent Medicine, \\ University of Ulm, Ulm, Germany
}

\begin{abstract}
The literature on obesity and weight regulation published in the last 12 months demonstrates the activity and interest of this field of science and medicine. For the Yearbook selection this year, only articles published in journals with a high impact factor were considered, assuming that relevant findings were published in these journals. The highlights are papers about new molecules involved in the regulation of eating and physical activity behavior as well as in addiction-like reward dysfunction and compulsive eating; papers on early programming of metabolic and endocrine regulation of body weight and papers demonstrating that the search for copy number variants (CNVs) are a new strategy to discover obesity genes which might contribute to the missing heritability of obesity.

In Yearbook 2009 the identification and importance of brown adipose tissue in adult humans has been demonstrated in three independent articles in the New England Journal of Medicine [1]. These findings have stimulated research in the molecular regulation of energy expenditure coming up with exciting new data presented in this year's volume.

In Yearbook 2007, two papers published in Nature showed for the first time that gut microbiota are a contributing factor to the pathophysiology of body weight regulation [2]. This story goes on as shown in an article published in Science, 2010.
\end{abstract}

\section{Adipose tissue \\ New molecule responsible for preadipocyte determination}

\section{Transcriptional control of preadipocytes determination by Zfp423}

Gupta RK, Arany Z, Seale P, Mepani RJ, Ye L, Conroe HM, Roby YA, Kulaga H, Reed RR, Spiegelman BM

Department of Cancer Biology and Division of Metabolism and Chronic Disease, Dana-Farber Cancer Institute and

Department of Cell Biology, Harvard Medical School, Boston, Mass., USA

Nature 2010;464:619-623

Background: Obesity is increasing in an epidemic manner. The size of the adipose tissue mass is determined by the number of fat cells as well as their volume. It is important to understand which mechanisms control the development of fat cells. The differentiation of committed preadipocytes to adipocytes is controlled by PPAR $\gamma$ and other transcription factors. However, the molecular basis for preadipocyte determination is not understood.

Methods: The authors used a new method for the quantitative analysis of transcriptional components ('Quanttrx') to identify new proteins involved in preadipocyte commitment. Results were confirmed using genetic approaches (ectopic expression, shRNA).

Results: The zinc-finger protein Zfp423 was identified as a protein that is enriched in preadipose versus non-preadipose fibroblasts. Expression of $\mathrm{Zfp} 423$ in non-preadipose fibroblasts activates PPAR $\gamma$ expression and permits cells to undergo adipocyte differentiation under permissive conditions. Knockdown of Zpf423 in preadipose 3T3-L1 cells blunts PPAR $\gamma$ expression and diminished the ability for adipogenic differentiation.

Conclusion: This study identifies Zpf423 as a transcriptional regulator of preadipocyte determination and suggests a model in which the regulation of the preadipocyte levels of PPAR $\gamma$ by Zpf423 represents a crucial determinant of preadipocyte commitment.

There is a high turnover of fat cells in human white adipose tissue [3]. Additionally, the white adipose tissue keeps the ability to expand throughout adult life by an increase in adipocyte number [4]. For a 
better understanding of the dynamics of adipose tissue and development of obesity it will be crucial to elucidate the whole adipogenic process - from stem cell precursor, via preadipose fibroblast to the mature adipocyte. The transcriptional pathways controlling adipocyte differentiation from committed preadipose fibroblasts were intensely investigated and there has been great progress. However, the transcriptional mechanisms underlying the preceding steps, the commitment of the embryonic stem cell precursor to the adipocyte lineage, remain to be characterized. The major problem seems to be that the preadipose commitment is more a quantitative than a qualitative trait [5]. Till now there is no specific marker which enables researchers to separate preadipose fibroblast from non-adipogenic fibroblast. In the present paper, for the first time, the authors were able to identify one protein that seems to be the important determinant - Zfp423. The authors showed in cell culture and in vivo (Zpf423 knockout mice) studies a genetic requirement for Zfp423 in the initial formation of both brown and white adipocytes. Taken together, this protein might help to gain more insight into the molecular basis of adipose tissue formation in physiological and pathophysiological states, thereby helping to provide new means to develop therapeutic strategies for the treatment and prevention of obesity.

\section{New molecules involved in the regulation of eating and physical activity behavior}

\section{Regulation of adaptive behavior during fasting by hypothalamic Foxa2}

Silva JP, von Meyenn F, Howell J, Thorens B, Wolfrum C, Stoffel M

The Rockefeller University, Laboratory of Metabolic Diseases, New York, N.Y., USA

Nature 2010;462:646-651

Background: Neurons in the lateral hypothalamus are important for feeding, behavioral arousal and addiction. These behaviors are stimulated by orexin and melanin-concentrating hormone (MCH). The aim of the study was to further understand the responsible molecular mechanisms.

Methods: Mouse and metabolic studies were performed including nuclear translocation experiments, laser scanning confocal microscopy, transfection and transactivation assays, and knockout mice.

Results: Forkhead box transcription factor Foxa2 is a downstream target of insulin signaling and is expressed in two distinct neuronal cell populations, namely $\mathrm{MCH}$ and orexin neurons. In the absence of insulin signaling, Foxa2 is recruited to the $\mathrm{MCH}$ and orexin promoters to activate their expression. Accordingly, during fasting resulting in low insulin levels Foxa2 binds to $\mathrm{MCH}$ and orexin promoters and stimulates their expression. By contrast, in the presence of insulin these two neuropeptides are down-regulated due to a change in the subcellular localization of Foxa2 which is regulated by plasma insulin concentrations. In fed and in hyperinsulinemic obese mice, Foxa2 is phosphorylated by insulin/ PI3K/Akt signaling which leads to Foxa2 inactivation and nuclear exclusion and finally to a reduced expression of $\mathrm{MCH}$ and orexin.

Constitutive nuclear activation of Foxa2 simulating the situation in the low insulinemic state in the brain results in increased $\mathrm{MCH}$ and orexin expression and increased food consumption, metabolism, and also in peripheral insulin sensitivity. In addition, spontaneous physical activity is significantly increased.

Conclusion: These data show that Foxa2 is a metabolic sensor in the lateral hypothalamus and integrates metabolic signals, adaptive behavior, and physiological responses.

Foxa2 in melanin-concentrating hormone- and orexin-expressing neurons is permanently inactivated in hyperinsulinemic mice with diet-induced obesity [6]. The authors show that insulin down-regulates Foxa2 expression by changing the subcellular localization of Foxa2 which is excluded from the nucleus by insulin. Hence, these studies show that there is a molecular explanation for the inverse associations of hyperinsulinemia/insulin resistance and physical activity/energy expenditure which has been observed in humans in several studies.

Interestingly, the authors could show convincingly that constitutive nuclear activation of brain Foxa2 mimicking hypoinsulinemia results in increased neuronal melanin-concentrating hormone and orexin expression in obese mice and leads to increased food consumption, improved glucose homeostasis, 
decreased fat and increased lean body mass linked to an increase in physical activity in these obese animals. Pharmacological inhibition of Foxa2 phosphorylation may be a possibility to increase the levels of physical activity, overall health and longevity.

\title{
Dopamine D2 receptors in addiction-like reward dysfunction and compulsive eating in obese rats
}

\author{
Johnson PM, Kenny PJ
}

Laboratory of Behavioral and Molecular Neuroscience, Department of Molecular Therapeutics, The Scripps Research Institute, Fla., USA

pjkenny@scripps.edu

Nature Neurosci 2010;13:635-641

Background: Food consumption is influenced by pleasure and reward. Obtaining food reward is a drive highly motivating food consumption. The hedonic mechanisms behind these observations which also might contribute to obesity remain poorly understood. The aim of the study was to investigate the effects of extended access to a palatable high-fat diet on the sensitivity of brain reward systems in rats. In addition, the role of striatal dopamine D2 receptors (D2R) in the resulting addiction-like behavioral responses was investigated.

Methods: Studies were performed with Wistar rats. Methods included bipolar stimulating of the lateral hypothalamus by an electrode, training of animals in a current-threshold brain stimulation reward (BSR) procedure, and lentivirus-mediated knockdown of striatal D2R expression.

Results: In the BSR procedure, rats respond vigorously to obtain rewarding electrical self-stimulation through the indwelling stimulating electrode, with the minimal stimulation intensity that maintains self-stimulation behavior termed the reward threshold. The development of obesity in rats with extended access to a cafeteria style diet consisting of palatable energy dense food was closely associated with a worsening deficit in brain reward function, reflected in progressively elevated BSR thresholds. These animals showed addiction-like reward deficits, overeating and loss of homeostatic energy balance. By contrast, restricted access to palatable food gave rise to binge-like patterns of consumption, but did not disrupt the brain reward function. Animals with extended food access took twice as much energy as compared to the restricted-access animals. Striatal expression of the D2R was lower in the extendedaccess rats than in the restricted-access or chow-only fed rats. There was also a clear inverse relationship between body weight and striatal D2R expression. There was a persistent reward dysfunction and hypophagia during abstinence in rats with extended access to a cafeteria diet. Knockdown of striatal D2R increased vulnerability to reward dysfunction in rats with extended access to a cafeteria diet. Knockdown of striatal D2 receptors markedly accelerated compulsive-like eating of palatable food. This was only observed in rats with a history of extended access to palatable food.

Conclusion: These data demonstrate that overconsumption of palatable food triggers addiction-like neuroadaptive responses in brain reward circuits and raises the development of compulsive eating.

Feeding is influenced by pleasure and reward. Easy access to palatable high-fat food is considered to be an important environmental risk factor for obesity [7]. The authors show that overstimulation of brain reward systems through excessive consumption of palatable, energy-dense food induce a profound state of reward hyposensitivity and the development of compulsive-like eating. The maladaptive behavioral responses in obese rats probably arise from diet-induced deficits in striatal D2 receptor signaling. Similarly, substance abuse decreases striatal D2R density, induces a profound state of reward hypofunction and triggers the emergence of compulsive-like drug-taking behaviors. Thus, the findings of the present paper support previous work [7-22] indicating that obesity and drug addiction may arise from similar neuroadaptive responses in brain reward circuits.

Conversely, individuals with anorexia nervosa have elevated striatal D2R. Weight loss after bariatric surgery is associated with elevated striatal D2R density [23, 24]. The gene polymorphism referred to as the TaqIA A1 allele results in decreased striatal D2R density, and individuals harboring this allele are over-represented in obese population [8].

So far the hedonic mechanisms contributing to obesity remain poorly understood. Interestingly in hyperphagic humans with congenital leptin deficiency, activity in the dorsal and ventral striatum, which are core components of brain reward circuits, increases markedly in response to images of food. Leptin replacement therapy attenuates both striatal activity and self-reported 'liking' of food 
[25]. These data already suggest that the striatum is important in hedonic aspects of feeding behavior. It also has been shown that activation of the striatum in response to highly palatable food is blunted in obese individuals when compared with lean controls [8].

Extended access to palatable high-fat food can induce addiction-like deficits in rat brain reward function. This might be an important source of motivation that may drive overeating and contribute to the development of obesity.

\section{Regulation of energy homeostasis by bombesin receptor subtype-3: selective receptor agonists for the treatment of obesity}

Guan X-M, Chen H, Dobbelaar PH, Dong Y, Fong TM, Gagen K, Gorski J, He S, Howard AD, Jian T, Jiang M, Kan Y, Kelly TM, Kosinski J, Lin LS, Liu J, Marsh DJ, Metzger JM, Miller R, Nargund RP, Palyha O, Shearman L, Shen Z, Stearns R, Strack AM, Stribling S, Tang YS, Wang S-P, White A, Yu H, Reitman ML

Merck Research Laboratories, Rahway, N.J., USA

Xiaoming_guan@merck.com, marc_reitman@merck.com

Cell Metabolism 2010;11:101-112

Background: Pharmacotherapy of obesity has limited success so far, due to the existence of multiple redundant and compensatory pathways in energy homeostasis. Bombesin receptor subtype-3 (BRS-3) is primarily expressed in the brain but its biological functions are not yet well investigated. Its natural ligands are unknown. The aim of the present study was to develop potent BRS-3 agonist and antagonist ligands that are suitable for exploring the role of BRS-3 in the regulation of food intake, metabolic rate, and body weight.

Methods: Rat and mice studies were performed including metabolic rate measurements and binding assays. Studies were also performed using several knockdown animals.

Results: A BRS-3 antagonist ligand increases food intake, body weight, and adipose tissue mass. A BRS-3 agonist reduces food intake and increases metabolic rate. BRS-3 binding sites were identified in the hypothalamus, caudal brainstem, and several midbrain nuclei. Prolonged high levels of receptor occupancy increased weight loss, suggesting a lack of tachyphylaxis. BRS-3 agonist effectiveness was maintained in several knockdown animals $\left(\mathrm{Npy}^{-/}\right.$, Agrp ${ }^{-/}, \mathrm{Mc}^{-\mathrm{r}^{-/}}, \mathrm{Cnr}^{-/-}$and $\mathrm{Lepr}^{\mathrm{db} / \mathrm{db}}$ mice). BRS-3 null mice lost weight upon treatment with either a MC4R agonist or a CB1R inverse agonist.

Conclusion: This study shows that BRS-3 has an important role in energy homeostasis, complementary to several well-known pathways. BRS-3 agonists may be a potential approach for the treatment of obesity.

Bombesin receptor substrate 3 (BRS-3) is a G-protein-coupled receptor whose natural ligand is unknown. In the present study the authors developed potent, selective agonist and antagonist ligands to explore BRS-3 function. Pharmacological activation of BRS-3 presents a potential therapy for obesity. Since the data also show that the regulation of energy homeostasis by BRS-3 is complementary to the Mc4R and CB1R pathways it could be suggested that such potential therapies involving BRS-3 could also be performed in combination with the pharmacological modulation of other pathways.

Food intake is under the control of both homeostatic and non-homeostatic (e.g. hedonic) mechanisms. The study supports the concept that natural BRS-3 ligands are peptides that signal BRS-3 sites at least in part in the hypothalamus. These natural ligands probably regulate energy supplies and/or hedonic drive by reaching. The precise mechanisms, including neuron types and pathways, by which BRS-3 signaling regulates energy metabolism, remain unclear. These mechanisms however seem to be complementary to the action of other factors regulating energy metabolism such as leptin, melanocortin, neuropeptide $Y$, and endocannabinoid. 


\title{
Signaling through Tyr985 of leptin receptor as an age/diet-dependent switch in the regulation of energy balance
}

\author{
You J, Yu Y, Jiang L, Li W, Yu X, Gonzalez L, Yang G, Ke Z, Li W, Li C, Liu Y \\ Key Laboratory of Nutrition and Metabolism, Institute for Nutritional Sciences, Shanghai Institutes for Biological \\ Sciences, and Graduate School of the Chinese Academy of Sciences, Shanghai, China \\ liuy@sibs.ac.cn \\ Mol Cell Biol 2010;30:1650-1659
}

Background: Diminished leptin signaling occurs in association with aging or feeding a high-fat diet. The aim of the present study is to identify the exact physiological mechanisms linking the environmental factors to the impairment in the leptin-mediated regulation of energy metabolism.

Methods: Knock-in line of mice (Y985F) expressing a mutant Ob-Rb, the long form of the leptin receptor with a phenylalanine substitution for $\mathrm{Tyr}^{985}$, one of the three intracellular tyrosines that mediate leptin's signaling actions.

Results: Surprisingly, whereas young homozygous Y985F animals were slightly leaner, they exhibit adultonset or diet-induced obesity. The observed age-dependent or diet-induced deterioration of energy balance was paralleled with pronounced leptin resistance. This leptin resistance was mainly attributable to attenuation of leptin-responsive hypothalamic STAT3 activation and elevated expression of hypothalamic SOCS3.

Conclusion: The study shows that there are distinct binary roles for $\mathrm{OBR} \mathrm{Tyr}^{985}$-mediated signaling in energy metabolism. These roles act as an age/diet-dependent regulatory switch to counteract age-associated or diet-induced obesity.

Leptin is an adipose-secreted hormone that plays a pivotal role in the regulation of energy metabolism. Acting through its receptor in distinct classes of leptin-responsive neurons, leptin activates multiple signaling pathways in the hypothalamus to regulate food intake and energy expenditure. The study shows here that the loss of $\mathrm{Tyr}^{985}$ in the long form of the leptin receptor (Ob-Rb) leads to adult onset of obesity. Adult Y985F mice exhibit impairments in both food intake and energy expenditure and a loss of Tyr ${ }^{985}$-mediated actions that exacerbates high-fat diet-induced obesity. Altogether this animal model confirms the role of the leptin receptor in the regulation of fat stores and energy metabolism and shows that mild impairment of leptin receptor signaling can produce more subtle phenotypes than those observed in human and mice so far.

\section{Early programming of metabolic and endocrine regulation of body weight}

\section{Early overnutrition results in early-onset arcuate leptin resistance and increased sensitivity to high-fat diet}

Glavas MM, Kirigiti MA, Xiao XQ, Enriori PJ, Fisher SK, Evans AE, Grayson BE, Cowley MA, Smith MS, Grove KL Oregon National Primate Research Center, Oregon Health and Science University, Beaverton, Oreg., USA Endocrinology 2010;151:1598-1610

Background: Childhood obesity is rising at an alarming rate, but we are far from understanding all mechanisms behind this trend. Reducing the size of a mice litter and exposing thereby mice to chronic postnatal overnutrition (CPO) is an interesting animal model to analyze mechanisms and consequences of early overfeeding.

Methods: Litters from Swiss Webster dams were culled to 3 pups (CPO) or 10 pups (control). On the 23 rd postnatal day all were weaned onto standard chow. At an age of 6 weeks, a subset of mice was placed on high-fat diet. In all subgroups, glucose and insulin tolerance were examined at 16-17 weeks of age. At postnatal 16 and in adulthood, leptin levels were measured and leptin sensitivity was determined by measurement of food intake and hypothalamic phosphorylated signal transducer and activator of transcription-3 (STAT3) immunoreactivity after intraperitoneal leptin injection.

Results: Compared to control pups, CPO mice exhibited an increased body weight and hyperleptinemia in the pre-weaning period, but only a slightly heavier body weight and normal glucose tolerance in 
adulthood on standard chow. Throughout their whole life however, CPO mice demonstrated an attenuated clinical leptin-responsiveness and a reduced STAT3 activation in the nucleus arcuatus, despite normalized leptin levels. In response to high-fat diet, CPO mice gained weight more rapidly and in contrast to control mice displayed a pathological response to an insulin tolerance test.

Conclusion: Early overfeeding leads to early and persistent leptin resistance and increases the susceptibility for overweight and insulin resistance.

\title{
Effects of maternal surgical weight loss in mothers on intergenerational transmission of obesity
}

\author{
Smith J, Cianflone K, Biron S, Hould FS, Lebel S, Marceau S, Lescelleur O, Biertho L, Simard S, Kral JG, Marceau, P \\ Institut Universitaire de Cardiologie et Pneumologie de Quebec, Laval University, Quebec, Canada \\ J Clin Endocrinol Metab 2009;94:4275-4283
}

Background: Maternal pre-pregnancy weight, pregnancy weight gain and metabolic derangements during pregnancy influence offspring obesity rate and co-morbidities. Pre-conceptive biliopancreatic diversion bariatric surgery provides a highly specific model to study the transmission of metabolic risk factors in humans by comparing children born before (BMS) and after maternal surgery (AMS).

Methods: Anthropometry and fasting blood levels were studied in 49 women who had lost $36 \pm 1.8 \%$ body weight and sustained this weight-loss for $12 \pm 0.8$ years and in their 111 children (54 BMS and 57 AMS) aged 2.5-26 years.

Results: AMS children had a lower birth weight than BMS children $(2.9 \pm 0.1$ vs. $3.3 \pm 0.1 \mathrm{~kg}, \mathrm{p}=0.003)$ and a lower prevalence of macrosomia ( 1.8 vs. $14.8 \%, \mathrm{p}=0.03)$. At the time of follow-up, AMS children showed a lower prevalence of obesity compared to BMS children (11 vs. $35 \%, p=0.004)$. Furthermore, AMS children had a higher insulin sensitivity (HOMA index $3.4 \pm 0.3$ vs. $4.8 \pm 0.5, p=0.02$ ) improved lipid profile (cholesterol/high-density lipoprotein cholesterol $2.96 \pm 0.11$ vs. $3.40 \pm 0.18, p=0.03$; highdensity lipoprotein cholesterol $1.50 \pm 0.05$ vs. $1.35 \pm 0.05 \mathrm{mmol} / \mathrm{l}, \mathrm{p}=0.04)$, lower C-reactive protein $(0.88 \pm 0.17$ vs. $2.00 \pm 0.24 \mu \mathrm{g} / \mathrm{ml}, \mathrm{p}=0.004)$, and leptin $(11.5 \pm 1.5$ vs. $19.7 \pm 2.5 \mathrm{ng} / \mathrm{ml}, \mathrm{p}=0.005)$ and increased ghrelin $(1.28 \pm 0.06$ vs. $1.03 \mathrm{ng} / \mathrm{ml} \pm 0.06)$ than BMS offspring.

Conclusion: Improved intrauterine environment via pre-conceptive bariatric surgery not only lowers the risk of childhood/adolescence obesity but also reduces accompanying cardio-vascular risk factors.

These two studies address the interesting topic of the influence of metabolic and endocrine programming on the development of obesity and co-morbidities, albeit in two completely different approaches.

The first group used an animal model of early overnutrition which leads to an increased susceptibility to overweight and hyperinsulinemia [26-28]. It has already been demonstrated that in postnatally overfed rats leptin shows a decreased inhibition of neuronal activity in the arcuate nucleus [29]. The reduced effect of leptin and a reduced induction of STAT3 expression seen here further strengthens the hypothesis that early leptin resistance might play an integral part in raising the long-term risk of obesity and metabolic derangements.

Men are not mice and whereas in rodents the neuronal network responsible for food intake regulation is progressively established during early postnatal life, in humans the major part of neuronal development occurs before birth [30]. Therefore, the mechanism by which postnatal overfeeding in mice enhances the risk of adulthood obesity and insulin resistance might essentially be comparable to the consequences of human obesity during pregnancy.

The study group of Kral et al. [31] is the first to examine the long-term impact of maternal weight loss through bariatric surgery on obesity rate and co-morbidity in offspring. In a previous study they were able to show an impressive reduction in childhood/adolescence obesity. Now they completed this examination by showing an associate improvement in cardiometabolic risk markers which was sustained into adolescence and young adulthood. This proves elegantly the influence of potentially modifiable epigenetic factors on childhood obesity and associated morbidity. Apart from the academic interest of this finding, it also has important practical implications. Preconceptive bariatric surgery decreases rates of gestational diabetes and neonatal macrosomia [32-35], however it increases the risk for internal hernias $[32,36,37]$ and severe nutritional deficiencies [38] during pregnancy. Therefore, each decision for bariatric surgery has to be made cautiously, but this study definitely strengthens the recommendation of bariatric surgery prior to conception in severely obese women. 
Leptin resistance cannot be evaluated easily in children. However, the lower leptin levels in BMS children point to the fact that early development of leptin resistance may be a major player of increased obesity susceptibility in humans as well as in rodents.

\section{Search for copy number variants (CNVs) \\ a new strategy to discover obesity genes}

\section{Large, rare chromosomal deletions associated with severe early-onset obesity}

Bochukova EG, Huang N, Keogh J, Henning E, Purmann C, Blaszczyk K, Saeed S, Hamilton-Shield J, Clayton-Smith J, O'Rahilly S, Hurles ME, Faroogi IS

University of Cambridge Metabolic Research Laboratories, Institute of Metabolic Science, Addenbrooke's Hospital, Cambridge, UK

Isf20@cam.ac.uk, meh@sanger.ac.uk

Nature 2010;463:666-670

Background: Genome-wide association studies have identified common single nucleotide polymorphisms (SNPs) associated with increased body mass index. However, together these account for a small percentage of inherited variations in BMI. In contrast, studies in patients with severe early-onset obesity have led to the detection of rare variants with significant impact on body weight. The aim of the present study was to explore the contribution of copy number variants (CNVs) to obesity.

Methods: 300 UK Caucasian patients with severe obesity defined as a BMI standard deviation score $>3$ and onset of obesity before 10 years of age were selected from the Genetics of Obesity Study Cohort. Of these individuals, 143 had developmental delay. DNA samples were run on Affymetrix genomewide human SNP arrays 6.0 by Aros, Inc.

Results: Large $(>500 \mathrm{~kb})$, rare $(<1 \%)$ deletions were significantly enriched in patients compared to $>7,000$ controls. Several CNVs were recurrent in patients but absent or at much lower prevalence in controls. Five patients with overlapping deletions on chromosome $16 \mathrm{p} 11.2$ were identified. In 3 patients the deletion co-segregated with severe obesity. In an independent sample of $>1,000$ patients with severe obesity alone, a 16p11.2 deletion was found in an additional 2 patients. All 16p11.2 deletions encompass several genes but include SH2B1, which is known to be involved in leptin and insulin signaling. Deletion carriers exhibited hyperphagia and severe insulin resistance disproportionate for the degree of obesity. Conclusion: The CNVs contribute significantly to the genetic architecture of human obesity.

\section{A new highly penetrant form of obesity due to deletions on chromosome 16p11.2}

Walters RG, Jacquemont S, Valsesia A, de Smith AJ, Martinet D, Andersson J, Falchi M, Chen F, Andrieux J, Lobbens S, Delobel B, Stutzmann F, El-Sayed Moustafa JS, Chevre JC, Lecoeur C, Vatin V, Bouquillon S, Buxton JL, Boute O, Holder-Espinasse M, Cuisset JM, Lemaitre MP, Ambresin AE, Brioschi A, Gaillard M, Giusti V, Fellmann F, Ferrarini A, Hadjikhani N, Campion D, Guilmatre A, Goldenberg A, Calmels N, Mandel JL, Le Caignec C, David A, Isidor B, Cordier MP, Dupuis-Girod S, Labalme A, Sanlaville D, Beri-Dexheimer M, Jonveaux P, Leheup B, Ounap K, Bochukova EG, Henning E, Keogh J, Ellis RJ, Macdermot KD, van Haelst MM, Vincent-Delorme C, Plessis G, Touraine R, Philippe A, Malan V, Mathieu-Dramard M, Chiesa J, Blaumeiser B, Kooy RF, Caiazzo R, Pigeyre M, Balkau B, Sladek R, Bergmann S, Mooser V, Waterworth D, Reymond A, Vollenweider P, Waeber G, Kurg A, Palta P, Esko T, Metspalu A, Nelis M, Elliott P, Hartikainen AL, McCarthy MI, Peltonen L, Carlsson L, Jacobson P, Sjostrom L, Huang N, Hurles ME, O'Rahilly S, Farooqi IS, Mannik K, Jarvelin MR, Pattou F, Meyre D, Walley AJ, Coin LJ, Blakemore Al, Froguel P, Beckmann JS

Section of Genomic Medicine, Department of Epidemiology and Public Health, Imperial College London, UK p.froguel@imperial.ac.uk, Jacques.beckmann@chuv.ch

Nature 2010;463:671-677

Background: Copy number variants (CNVs) might contribute to the missing heritability of common disorders and also of obesity. 
Methods: Subjects ascertained for cognitive deficit (malformations) with or without obesity were selected from those clinically referred for genetic testing. 16p11.2 deletions were identified in these individuals by standard clinical diagnostic procedures. Algorithmic analyses of GWAS data were performed.

Results: A highly penetrant form of obesity was initially observed in 31 subjects who were heterozygous for deletions at 16p11.2 and who had also cognitive deficits. Nineteen similar deletions were identified from GWAS data in $>16,000$ individuals from eight European cohorts. These deletions were absent from healthy non-obese controls and accounted for $0.7 \%$ of the morbid obesity cases (BMI $\geq 40$ or BMI-SDS $\geq 4$ ).

Conclusion: These data demonstrate the potential importance of rare variants with strong effects in common diseases as it could be shown here for a CNV in 16p11.2 for obesity.

These two studies show a promising strategy for identifying missing heritability in obesity (and other complex traits). Cohorts with extreme phenotypes are likely to be enriched for rare variants, with increased chance of discovery. The loci identified by this strategy may be subsequently analyzed and reveal additional rare variants that further contribute to the missing heritability. It seems to be very productive to combine 'the power of the extreme' [40]: small, well-phenotyped cohorts with targeted follow-up and large population cohorts. Strategies aimed at looking for rare variants near common susceptibility loci may well prove to be fruitful in other common complex disease.

The study by Bochukova et al. showed that a deletion of $16 \mathrm{p} 11.2$ is associated with highly penetrant familial severe early-onset obesity and severe insulin resistance. The data suggest that although the contribution of other genes or non-coding genetic material cannot be excluded, the phenotype is consistent with a role for SH2B1 in human energy homeostasis and glucose metabolism. The prevalence of the SH2B1 containing deletion in patients with severe early-onset obesity was significantly greater than in controls. SH2B1 encodes an adaptor protein involved in leptin and insulin signaling. Disruption of SH2B1 in mice results in obesity and severe insulin resistance [39].

\section{Molecular regulation of energy expenditure}

\section{Sarcolemmal ATP-sensitive $\mathrm{K}^{+}$channels control energy expenditure determining body weight}

Alekseev AE, Reyes S, Yamada S, Hodgson-Zingman DM, Sattiraju S, Zhu Z, SierraA, Gerbin M, Coetzee WA, Goldhamer DJ, Terzic A, Zingman LV

Department of Medicine, Mayo Clinic, Rochester, Minn., USA

Cell Metab 2010;11:58-69

Background: Energy-conserving mechanisms promote obesity in an environment of hyperalimentation and sedentary lifestyle. Comprehension of these mechanisms could help to interfere with their efficiency to advance obesity treatment and prevention. ATP-sensitive $\mathrm{K}^{+}$channels are involved in setting muscle energy expenditure.

Methods: Two mouse models of $\mathrm{K}_{\mathrm{ATP}}$ channel deficiency were characterized in terms of energy metabolism.

Results: The disruption of $\mathrm{K}_{\mathrm{ATP}}$ channel function raised energy expenditure in both cardiac and skeletal muscle, while locomotor activity and blood substrate availability were unaltered. As a consequence of this fuel metabolism inefficiency, glycogen and body fat depots were reduced, leading to a lean phenotype. Under high-fat diet, the phenotype of a reduced body weight of $\mathrm{K}_{\mathrm{ATP}}$ channel-deficient mice persisted. However, this obesity retardation was accompanied by a decreased workload endurance.

Conclusions: Downregulation of sarcolemmal $\mathrm{K}_{\mathrm{ATP}}$ channels could provide a novel option against obesity by interfering with muscle energy efficiency.

$\mathrm{K}_{\text {ATP }}$ channels are hetero-octameric proteins composed of inwardly rectifying $\mathrm{K}^{+}$channel (Kir6.x) and sulfonylurea receptor (SUR) subunits. Different combinations of Kir6.x and SUR subunits comprise $\mathrm{K}_{\text {ATP }}$ channels with distinct electrophysiological and pharmacological properties. Coupling of phos- 
photransfer pathways with $\mathrm{K}_{\text {ATP }}$ channels permits a transduction of nucleotide fluxes into changes in membrane excitability, matching energy demands with metabolic resources. $K_{\text {ATP }}$ channels are expressed in various tissues including heart, kidney, pancreatic $\beta$ cells, central neurons and skeletal and smooth muscle [41], there functioning as metabolic sensors assigned to protective responses under severe energy insult. Former studies with Kir6.2-deficient mice suggest a role for these channels in skeletal muscle glucose uptake [42]. The present study describes a novel role for sarcolemmal $\mathrm{K}_{\text {ATP }}$ channels under non-stressed, physiological conditions. Lack of these channels led to an increase in energy expenditure which resulted in the decrease of body glycogen and fat stores promoting a lean phenotype, even under high-fat diet. The authors propose that the protective effect of the downregulation of $K_{\text {ATP }}$ channels may be a novel option for obesity therapy. However, the described reductions in workload endurance will limit this approach, for instance by interfering with lifestyle intervention programs.

\title{
Fyn-dependent regulation of energy expenditure and body weight is mediated by tyrosine phosphorylation of LKB1
}

\author{
Yamada E, Pessin JE, Kurland IJ, Schwartz GJ, Bastie CC \\ Department of Medicine, Diabetes Research and Training Center, Albert Einstein College of Medicine, Bronx, N.Y., \\ USA \\ claire.bastie@einstein.yu.edu \\ Cell Metabolism 2010;11:113-124
}

Background: Fyn null mice show an increased fatty acid oxidation and energy expenditure resulting in a reduced adipose tissue mass and enhanced insulin sensitivity. Fyn is a negative regulator of fatty acid oxidation through inhibition of the AMP-dependent protein kinase (AMPK). AMPK is regarded as an energy sensor. It is regulated directly by the intracellular AMP/ATP ratio. In a state of caloric excess, AMPK is inactive, resulting for instance in an increased fatty acid storage. An upstream kinase activator of AMPK in peripheral tissue is LKB1 which is inactive as long as it is localized in the nucleus.

Methods: The authors inhibited Fyn kinase with SU6656 in WT mice. They determined whole-body energy expenditure via indirect calorimetry and total fat mass by magnetic resonance. Fatty acid oxidation was assessed in isolated skeletal muscles. Additionally, they treated murine muscle and adipocyte cell lines with SU6656 followed by localization studies of LKB1. Validation experiments were performed with genetic approaches.

Results: Selective pharmacological inhibition of Fyn in wild-type mice resulted in a similar phenotype as Fyn-null mice (increased fatty acid oxidation, elevated energy expenditure, lean). The authors showed that Fyn-dependent phosphorylation of LKB1 is responsible for the nuclear localization of LKB1. Inhibition of Fyn kinase results in a reduced rate of nuclear import of LKB1. A higher amount of LKB1 in the cytoplasm leads to activation of AMPK, thereby increasing the fatty acid oxidation and the energy expenditure.

Conclusion: The positive metabolic effects seen in Fyn-null mice (decreased adiposity, increased energy expenditure) can be reproduced by the acute pharmacological inhibition of Fyn activity. These data highlight the therapeutic potential of inhibiting Fyn kinase signaling for obesity and its related disorders.

By inhibiting the Fyn kinases, an important player in energy metabolism, the authors showed an increased energy expenditure and increased fatty acid oxidation leading to a decreased fat mass in mice. These effects are similar to those they already observed in a knockout mouse for Fyn [43]. The finding of Yamada and colleagues' suggest that this enzyme which belongs to the Src family of nonreceptor tyrosine kinases might offer a useful target for obesity therapy. However, the inhibitor SU6656 used in the present study is not a drug candidate. Both Fyn kinase as well as the downstream target of Fyn, AMPK, are important in the brain besides their action in fat and muscle. Therefore, it will be necessary to find something acting only on adipose tissue and muscle. Another possible way might be to manipulate the Fyn kinase gently with nutrients. For instance, epigallocatechin gallate which can be found in green tea inhibits Fyn kinase [44] and mimics thereby the action of the SU6656. Green tea has already been associated with weight loss which was explained by thermogenic effects probably partly due to the caffeine in the green tea [45-47]. The role of Fyn in this context has now to be considered. Taken together, this publication shows a new possible target for obesity therapy - the Fyn kinase. But due to its important role in the brain as well, more research has to be done. It will be essential to map 
all regulatory elements of this signaling pathway to identify its regulatory actions and to elucidate how this pathway functionally integrates with other pathways to further support the potential of Fyn as a drug target for the treatment of obesity.

\title{
Gs $\alpha$ deficiency in adipose tissue leads to a lean phenotype with divergent effects on cold tolerance and diet-induced thermogenesis
}

\author{
Chen M, Chen H, Nguyen A, Gupta D, Wang J, Lai EW, Pacak K, Gavrilova O, Quon MJ, Weinstein S \\ Metabolic Diseases Branch, National Institute of Diabetes and Digestive and Kidney Diseases, \\ National Institutes of Health, Bethesda, Md., USA \\ Cell Metabolism 2010;11:320-330
}

Background: $\mathrm{G}_{\mathrm{s}}$, a G-protein-mediating receptor-stimulated cAMP production, is involved in adipogenesis and adipose tissue function. Mutations of this protein lead to an obesogenic phenotype in both mice and patients with Albright hereditary osteodystrophy (AHO).

Methods: An adipose-specific $\mathrm{G}_{\mathrm{s}}$-deficient mouse model was generated and analyzed in terms of adipose function and thermogenic properties.

Results: Heterozygeous animals did not show an obvious phenotype, indicating that obesity seen in AHO is not caused by an adipose-specific $\mathrm{G}_{\mathrm{s}}$ deficiency. Homozygotes displayed a strong reduction of adipose tissue, pointing to a role of $G_{s}$ in adipogenesis. This came along with impaired cold tolerance and reduced brown adipose tissue (BAT) response to sympathetic stimuli. However, diet-induced thermogenesis and fatty acid oxidation in skeletal muscle were increased. Under high-fat diet, wild-type mice displayed enhanced sympathetic nerve activity in muscle, but not in BAT.

Conclusion: The results of this study indicate that cold- and diet-induced thermogenesis occurs in different tissues. According to these data, BAT is not the tissue responsible for diet-induced thermogenesis.

Brown adipose tissue (BAT) is the site of active glucose uptake in humans as recently demonstrated by PET/CT imaging. This has led to a renewed interest in the function of BAT thermogenesis in the regulation of body weight. Diet-induced thermogenesis (DIT) results from increased energy expenditure secondary to changes in nutrient uptake and is partly mediated by the sympathetic nervous system (SNS). SNS induced thermogenesis in BAT is partially mediated by $G_{s} \alpha$, a ubiquitously expressed $G$ protein which couples hormone and neurotransmitter receptors to the generation of intracellular ATP. In the present study, the authors investigated the participation of this protein in thermogenesis in a $G_{s} \alpha$-deficient mouse model. These mice were cold-intolerant due to disruption of SNS signaling to BAT indicating a defect in cold-induced thermogenesis. Under high-fat diet, DIT was paradoxically increased in knock-out mice. In the absence of BAT thermogenesis this indicates that another tissue must be responsible for DIT, suggestively the skeletal muscle.

DIT is due to an increase in energy expenditure after changes in nutrient uptake. So far, it was thought to result from adrenergic stimulation of BAT and to be mediated by BAT uncoupling protein UCP-1 [48]. However, UCP-1-ablated mice do not show an obesogenic phenotype unless they are housed at physiological temperatures [49,50]. Altogether, this model questions the involvement of BAT tissue in DIT and points to additional tissue sites involved. To further confirm these data, studies with human brown adipocytes ex vivo are needed. The mechanisms for obesity in $\mathrm{G}_{\mathrm{s}} \alpha$ deficiency in humans remain elusive.

\section{Initiation of myoblast to brown fat switch by a PRDM16-C/EBP- $\beta$ transcriptional complex}

\author{
Kajimura S, Seale P, Kubota K, Lunsford E, Frangioni JV, Gygi SP, Spiegelman BM \\ Dana-Farber Cancer Institute, Department of Cell Biology, Harvard Medical School, Division of Hematology/ \\ Oncology, Beth Israel Deaconess Medical Center, Mass., USA \\ Nature 2009;460:1154-1159
}

Background: Recent publications by this group identified PRDM16 (PR domain zinc finger protein 16) as a factor of brown fat determination. It stimulates brown fat-selective gene expression while suppressing the expression of genes selective for white fat cells. Brown adipocytes arise from precursors expressing the myoblast lineage marker Myf5. However, the mechanism of this determination is currently unknown. 
Methods: The authors identified putative binding partners of PRDM16 by proteomic analysis of transcriptional complexes formed with wild-type and differentiation-incompetent mutant PRDM16. Subsequently, the expressions of these candidates in white and brown adipose tissue were analyzed and their function in the process of myoblast to brown fat conversion by PRDM16 was investigated.

Results: Proteomic analyses and expression profiling revealed C/EBP- $\beta$ as a binding partner of PRDM16. Overexpression of both factors in fibroblastic cells including mouse and human skin fibroblasts was sufficient to induce a fully functional brown fat program. Transplants of fibroblasts expressing both PRDM16 and C/EBP- $\beta$ into mice led to the development of a fat pad with morphological and biochemical characteristics of brown adipose tissue. By using PET-CT scans, this fat pad was shown to act as a sink for glucose similar to endogenous brown adipose tissue.

Conclusions: The data show that the induction of brown fat is under the control of PRDM16 by formation of a transcription complex with C/EBP- $\beta$. This might offer novel therapeutic opportunities for obesity or type 2 diabetes.

In mammals, there are two types of adipose tissue with distinct functions. While white adipose tissue is the main storage organ of the body, brown adipose tissue regulates the body temperature by dissipating energy in the form of heat. This process is driven by uncoupling mitochondrial respiratory chain from ATP production by the protein UCP-1 (uncoupling protein 1). Since recruitment of brown adipose tissue is currently discussed as a strategy to eliminate excess energy in obesity, it is important to understand the molecular mechanisms of brown fat cell determination.

Recent studies suggest different origins of brown and white adipose tissue. Atit et al. [51] proposed a common lineage for muscle and brown fat on the basis of their finding that specific Myf5-expressing cells of the dermomyotome give rise to both muscle and brown fat cells but not to white adipocytes. Beneath several transcriptional regulators, PRDM16 was recently described as a switch in brown fat cell development by inducing brown adipocyte differentiation from white preadipocytes and myoblastic precursors [52]. However, disruption of DNA binding of PRDM16 does not interfere with its ability to induce a brown phenotype [53]. The authors suggested therefore that PRDM16 acts predominantly by protein-protein interactions rather than by DNA interaction. Indeed, PRDM16 coactivates the transcriptional regulators PGC- 1 and PPAR $\gamma$. By proteomic analyses, the authors could show here C/EBP- $\beta$ as a binding partner of PRDM16 which is involved in adipocyte development. This study provides a detailed insight into brown adipocyte determination and introduces a new potential target for enhancing brown adipose tissue in the context of obesity therapy.

\section{Gut microbiota \\ The story goes on}

\section{Metabolic syndrome and altered gut microbiota in mice lacking Toll-like receptor $\mathbf{5}$}

Vijay-Kumar M, Aitken JD, Carvalho FA, Cullender TC, Mwangi S, Srinivasan S, Sitaraman SV, Knight R, Ley RE, Gewirtz AT

Department of Pathology, Emory University, Atlanta, Ga., USA

agewirt@emory.edu

Science 2010;328:228-231

Background: Recent research has linked the mammalian host-gut microbial relationship to human obesity. There is growing evidence for a role of gut microbiota in both chronic inflammation and insulin resistance.

Methods: By using mice genetically deficient in Toll-like receptor 5 (TLR5), this study supports a direct relationship between impairment of the innate immune system, altered microbiota composition and development of metabolic syndrome. Mice embryos were transplanted into standard mice to standardize the microbiota in $\mathrm{KO}$ and control mice.

Results: Genetically deficient in TLR5, T5KO mice had a mild colitis and paradoxically, at 20 weeks of age, 20\% higher body mass index than WT mice. Serum triglycerides, cholesterol and blood pressure 
were increased compared to WT mice. In addition, a higher ex vivo production of proinflammatory cytokines such as interferon- $\gamma$ and interleukin- $1 \beta$ was observed in adipose tissue of T5KO than in WT mice. T5KO mice also exhibited hyperglycemia after fasting and an impaired glucose tolerance, elevated basal serum insulin levels and insulin resistance with high lipocalin-2 levels and increased number and size of pancreatic islets.

Metabolic syndrome developed in T5KO after administering an 8-week high-fat diet. In contrast to WT mice, which also showed significant increase in body mass, T5KO became diabetic with fasting blood glucose concentrations above $120 \mathrm{mg} / \mathrm{dl}$. They developed hepatic steatosis and inflammatory infiltrates in the pancreatic islets. The metabolic syndrome depends on hyperphagia since T5KO consume about $10 \%$ more food than WT littermates. Twelve weeks of food restriction prevented many of the metabolic abnormalities in $\mathrm{T} 5 \mathrm{KO}$ mice.

The metabolic syndrome in T5KO mice is independent of the adaptive immune system and TLR2/ TLR4, but results from the gut microbiota. The gut microbiota by broad-spectrum antibiotics in 4-week-old mice lowered the bacterial load by $90 \%$ and corrected food intake, fat pad and fasting glucose. Even the gut microbiota from T5KO mice intragastrically transferred in 4-week old WT germ-free mice induced elevated food intake, weight gain and lower insulin sensitivity with increase of inflammatory cytokine production in these mice.

Conclusion: Malfunctions of the innate immune system in TLR5-deficient mice alter the gut microbiota and contribute to the development of an increased fat mass and the metabolic syndrome in mice.

Nutritional and genetic factors alter the intestinal microbiota and may predispose individuals to the metabolic syndrome. The influence of gut microbiota on nutrient absorption and metabolic regulation has been demonstrated in human and animal studies showing alterations of the gut microbiota $[54,55]$. Obesity is associated with inflammation which might contribute to the increased risk of cardiovascular disease and type 2 diabetes. Innate immune dysfunction, such as Toll-like receptor deficiency, results in serum antibody production against commensal microbiota and disturbed host-commensal mutualism [56]. TLR5 is the main component of the innate immune system in the intestine. Surprisingly, Vijay-Kumar presented a TLR5 null mice phenotype with only mild colitis, but increased fat mass and typical signs of metabolic syndrome. This is the first link between malfunction of the innate immune system, changes in gut microbiota and the metabolic syndrome. Alterations in host-microbiota interactions may drive obesity and metabolic syndrome. Additional studies will show whether it is possible to change the microbiota in a way that ameliorates the metabolic profile in terms of food absorption or energy storage and hyperphagia.

\section{Bariatric surgery in adolescents}

\section{Laparoscopic adjustable gastric banding in severely obese adolescents: a randomized trial}

O'Brien PE, Sawyer SM, Laurie C, Brown WA, Skinner S, Veit F, Paul E, Burton PR, McGrice M, Anderson M, Dixon JB

FRACS, Centre for Obesity Research and Education, Monash University Medical School, Alfred Hospital, Melbourne, Vic., Australia

paul.obrien@med.monash.edu.au

JAMA 2010;303:519-526

Background: Adolescents with extreme obesity is a serious health challenge. Extreme obesity in adolescents is associated with both immediate and late health effects. Bariatric surgery has been shown to be the only effective treatment in obese adults leading to sizeable weight reduction. There are only a few observative studies in adolescents applying bariatric surgery. The aim of the study therefore was to compare the outcomes of gastric banding with an optimal lifestyle program in adolescent obesity.

Methods: Prospective, randomized, controlled study with 50 adolescents (age 14-18 years, BMI >35), follow-up visits during 2 years. Eligibility criteria included identifiable medical complications such as hypertension, metabolic syndrome, asthma, back pain, physical limitations such as an inability to play 
a sport, difficulties with activities of daily lining, or psychosocial difficulties such as isolation or low self-esteem and evidence of attempts to lose weight by lifestyle means more than 3 years.

Results: In the gastric banding group a weight loss of $34.6 \mathrm{~kg}(30.2-39.0)$ representing an excess weight loss of $79 \%$ and a BMI z score change from 2.4 to 1.3 was achieved. The mean weight loss in the lifestyle group was $3.0 \mathrm{~kg}(2.1-8.1)$ representing excess weight loss of $13 \%$ and a BMI z score change from 2.4 to 23 . In the gastric banding group a more favorable improvement in metabolic factors was observed as well as in quality of life. There were no perioperative adverse events. However, 8 operations $(33 \%)$ were required in 7 patients for revisional procedures either for proximal pouch dilatation or tubing injury during follow-up.

Conclusion: Gastric banding in obese adolescents resulted in much greater weight losses than lifestyle intervention and was associated with more favorable benefits to health and quality of life.

This study shows that gastric banding in obese adolescents proves to be an effective intervention leading to substantial and durable reduction of obesity and to better health. It should however be mentioned that the gastric banding approach to weight loss is not a quick fix. For optimal effectiveness, it requires long-term supportive follow-up by trained health professionals. The need for additional surgery for enlargement of the stomach above the band or injury to the tubing is intrinsic to the gastric banding procedure. Eating small meals slowly is central to avoiding this problem after the gastric banding procedure. For adolescents, additional education and supervision of eating are necessary to reduce the need for revisions.

Severe obesity in adolescents is associated with multiple serious diseases, impaired quality of life, and an increased risk for later cardiovascular and other diseases. This study confirms that lifestyle treatments can achieve weight loss and improvement in health for some individuals. Diligent application of these approaches should remain the first option for obese adolescents. However, the majority of patients do not respond to this approach. Therefore, laparoscopic adjustable gastric banding may be a future option for these patients. It also has been argued that adolescents with severe obesity need treatment during adolescence rather than deferring until adulthood.

In the context of the study it should be mentioned that the Endocrine Society has published guidelines for bariatric surgery in obese adolescents [1].

\section{Obesity prevention in schools No clarity about cost-effectiveness}

\section{The cost-effectiveness of Australia's active after-school communities program}

Moodie ML, Carter RC, Swinburn BA, Haby MM

Deakin Health Economics, Public Health Research Evaluation and Policy Cluster, Deakin University, Burwood, Vic.,

Australia

Marj.moodie@deakin.edu.au

Obesity (Silver Spring). 2009, Nov 5. Epub ahead of print

The school is an ideal place for obesity prevention programs. So-called 'after-school programs' are therefore reasonable because the time after school lessons is an optimal time to increase the physical activity within sport programs and simultaneous reduce the common consumption of sugar and fatty snacks at this time of day. For more than 5 years the Australian government has supported the so-called active-after-school-communities (AASC) program within a comprehensive national initiated program for obesity prevention. Cost-effectiveness analysis, conducted by health economists using simulation and modeling not described here showed that the program is not cost-effective as such. The authors suggest to improve the cost-effectiveness by increasing the duration of physical activity and raising the number of participating school children. 


\section{Minireview: Molecular targets for obesity therapy in the brain}

Obici S

University of Cincinnati, Obesity Research Center, Cincinnati, Ohio, USA

silvana.obici@uc.edu

Endocrinology 2009;150:2512-2517

This review summarizes recent progress in the identification of the neural pathways that respond to peripheral signals of energy availability such as leptin and macronutrients. The control of energy balance is achieved through neural pathways that receive metabolic signals. These pathways integrate these signals and activate behavioral and metabolic responses in order to maintain constant body weight and energy stores. These homeostatic mechanisms are mediated by neurons in the hypothalamus. In addition, other areas of the brain are involved in hedonic rewarding, and motivational aspects of ingestive behavior which contribute to modulate energy balance. This review summarizes recent progress in understanding the neural mechanisms by which leptin and nutrients modulate energy balance. Several molecular pathways recognized as general sensors of energy flux are emerging as pivotal sensors of energy availability in the hypothalamus. The convergence of nutrients and hormonal signals on a number of signaling pathways in the hypothalamus suggests that several nutritional cues are sensed and integrated to regulate energy balance. The disruption of the sensing and integration of these neural signals might be a crucial factor that leads to the development of obesity. Furthermore, these pathways represent a likely target for therapeutic intervention.

\section{Obesity and thyroid function}

Reinehr T.

Department of Paediatric Nutrition Medicine, Vestische Hospital for Children and Adolescents,

University of Witten/Herdecke, Datteln, Germany

T.Reinehr@kinderklinik-datteln.del

Mol Cell Endocrinol 2010;316:165-171

Advanced understanding of central mechanisms controlling energy homeostasis and data from clinical research characterizing hormonal and metabolic phenotypes of obese humans has renewed scientific interest in exploring the role of thyroid function in obesity. Elevated levels of thyrotropin (TSH) with concurrently normal thyroxine $\left(\mathrm{T}_{4}\right)$ and normal to slightly elevated levels of free triiodothyronine $\left(\mathrm{fT}_{3}\right)$ are a common finding in obese children and adolescents, as well as in adults. Nonetheless, cause and consequences of the obesity-associated state of 'subclinical hypothyroidism' have not been fully elucidated. Here, published data since the 1970 s on the impact of $\mathrm{T}_{3}$ on energy expenditure and $\mathrm{T}_{3}$ metabolism in states of over- and undernutrition are reviewed. Increased TSH and $\mathrm{fT}_{3}$ levels in obesity could be interpreted as an adaptational process increasing resting energy expenditure and therefore reducing the conversion of excess energy into fat stores. As a second focus, the review presents available data on the association of leptin and TSH levels and current hypotheses on possible interactions of TSH and leptin signaling. While identifying the exact molecular mechanisms linking obesity and thyroid function and learning how to manipulate thyroid hormone pathways is a rewarding task in obesity research, clinicians should be assured that at least thyroxine supplementation never has been - and probably never will be - a viable treatment option for obesity at any age.

\section{References}

1. Wabitsch M, Horenburg S, Denzer C, von Schnurbein J, Keuper M, Tews D, Moss A, Posovszky C, Fischer-Posovszky P: Obesity and weight regulation; in Carel J-C, Hochberg Z (eds): Yearbook of Pediatric Endocrinology. Basel, Karger, 1990.

2. Wabitsch M, Fuchs M, Horenburg S, Denzer C, von Puttkamer J, Moss A, Lahr G, Fischer-Posovszky P: Obesity and weight regulation; in Carel J-C, Hochberg Z (eds): Yearbook of Pediatric Endocrinology. Basel, Karger, 2007, pp 119132.

3. Spalding KL, Arner E, Westermark PO, Bernard S, Buchholz BA, Bergmann O, et al: Dynamics of fat cell turnover in humans. Nature 2008;453:783-787.

4. Prins JB, O’Rahilly S: Regulation of adipose cell number in man. Clin Sci (Lond) 1997;92:3-11.

5. Green H, Kehinde O: An established preadipose cell line and its differentiation in culture. II. Factors affecting the adipose conversion. Cell 1975;5:19-27. 
6. Byberg L, Zethelius B, McKeigue PM, Lithell HO: Changes in physical activity are associated with changes in metabolic cardiovascular risk factors. Diabetologia 2001;44:2134-2139.

7. Volkow ND, Wise RA: How can drug addiction help us understand obesity? Nat Neurosci 2005;8:555-260.

8. Stice E, Spoor S, Bohon C, Small DM: Relation between obesity and blunted striatal response to food is moderated by TaqIA A1 allele. Science 2008;322:449-452.

9. Noble EP: Addiction and its reward process through polymorphisms of the D2 dopamine receptor gene: a review. Eur Psychiatry 2000;15:79-89.

10. Wang GJ, Volkow ND, Fowler JS: The role of dopamine in motivation for food in humans: implications for obesity. Expert Opin Ther Targets 2002;6:601-609.

11. Booth ML, Wilkenfeld RL, Pagnini DL, Booth SL, King LA: Perceptions of adolescents on overweight and obesity: the weight of opinion study. J Paediatr Child Health 2008;44:248-252.

12. Puhl RM, Moss-Racusin CA, Schwartz MB, Brownell KD: Weight stigmatization and bias reduction: perspectives of overweight and obese adults. Health Educ Res 2008;23:347-358.

13. American Medical Association: Diagnostic and Statistical Manual of Mental Disorders, ed 4 (DSM-IV). Washington, American Psychiatric Association Publishing, 1994.

14. Markou A, Koob GF: Construct validity of a self-stimulation threshold paradigm: effects of reward and performance manipulations. Physiol Behav 1992;51:111-119.

15. Rolls BJ, Rowe EA, Turner RC: Persistent obesity in rats following a period of consumption of a mixed, high energy diet. J Physiol 1980;298:415-427.

16. Ahmed SH, Kenny PJ, Koob GF, Markou A: Neurobiological evidence for hedonic allostasis associated with escalating cocaine use. Nat Neurosci 2002;5:625-626.

17. Markou A, Koob GF: Postcocaine anhedonia. An animal model of cocaine withdrawal. Neuropsychopharmacology 1991;4:17-26.

18. Kenny PJ, Chen SA, Kitamura O, Markou A, Koob GF: Conditioned withdrawal drives heroin consumption and decreases reward sensitivity. J Neurosci 2006;26:5894-5900.

19. Cottone P, Sabino V, Steardo L, Zorrilla EP: Opioid-dependent anticipatory negative contrast and binge-like eating in rats with limited access to highly preferred food. Neuropsychopharmacology 2008;33:524-535.

20. Llado I, Estrany ME, Rodriguez E, Amengual B, Roca P, Palou A: Effects of cafeteria diet feeding on $\beta_{3}$-adrenoceptor expression and lipolytic activity in white adipose tissue of male and female rats. Int J Obes Relat Metab Disord 2000;24:1396-1404.

21. Fishburn CS, Elazar Z, Fuchs S: Differential glycosylation and intracellular trafficking for the long and short isoforms of the D2 dopamine receptor. J Biol Chem 1995;270:29819-29824.

22. Vanderschuren LJ, Everitt BJ: Drug seeking becomes compulsive after prolonged cocaine self-administration. Science 2004;305:1017-1019.

23. Wang GJ, Tomasi D, Backus W, Wang R, Telang F, Geliebter A, et al: Gastric distention activates satiety circuitry in the human brain. Neuroimage 2008;39:1824-1831.

24. Frank GK, Bailer UF, Henry SE, Drevets W, Meltzer CC, Price JC, et al: Increased dopamine D2/D3 receptor binding after recovery from anorexia nervosa measured by positron emission tomography and $\left[{ }^{11} \mathrm{C}\right]$ raclopride. Biol Psychiatry 2005;58:908-912.

25. Farooqi IS, Bullmore E, Keogh J, Gillard J, O’Rahilly S, Fletcher PC: Leptin regulates striatal regions and human eating behavior. Science 2007;317:1355.

26. Plagemann A, Harder T, Rake A, Waas T, Melchior K, Ziska T, et al: Observations on the orexigenic hypothalamic neuropeptide Y-system in neonatally overfed weanling rats. J Neuroendocrinol 1999;11:541-546.

27. Plagemann A, Heidrich I, Gotz F, Rohde W, Dorner G: Obesity and enhanced diabetes and cardiovascular risk in adult rats due to early postnatal overfeeding. Exp Clin Endocrinol 1992;99:154-158.

28. Rodrigues AL, de Moura EG, Passos MC, Trevenzoli IH, da Conceicao EP, Bonono IT, et al: Postnatal early overfeeding induces hypothalamic higher SOCS3 expression and lower STAT3 activity in adult rats. J Nutr Biochem 2010, Epub ahead of print.

29. Davidowa H, Plagemann A: Decreased inhibition by leptin of hypothalamic arcuate neurons in neonatally overfed young rats. Neuroreport 2000;11:2795-2798.

30. McMillen IC, Robinson JS: Developmental origins of the metabolic syndrome: prediction, plasticity, and programming. Physiol Rev 2005;85:571-633.

31. Kral JG, Biron S, Simard S, Hould FS, Lebel S, Marceau S, et al: Large maternal weight loss from obesity surgery prevents transmission of obesity to children who were followed for 2 to 18 years. Pediatrics 2006;118:e1644-e1649.

32. Patel JA, Patel NA, Thomas RL, Nelms JK, Colella JJ: Pregnancy outcomes after laparoscopic Roux-en-Y gastric bypass. Surg Obes Relat Dis 2008;4:39-45.

33. Wittgrove AC, Jester L, Wittgrove P, Clark GW: Pregnancy following gastric bypass for morbid obesity. Obes Surg 1998;8:461-464.

34. Ducarme G, Revaux A, Rodrigues A, Aissaoui F, Pharisien I, Uzan M: Obstetric outcome following laparoscopic adjustable gastric banding. Int J Gynaecol Obstet 2007;98:244-147.

35. Dixon JB, Dixon ME, O‘Brien PE: Birth outcomes in obese women after laparoscopic adjustable gastric banding. Obstet Gynecol 2005;106:965-972.

36. Marceau P, Kaufman D, Biron S, Hould FS, Lebel S, Marceau S, et al: Outcome of pregnancies after biliopancreatic diversion. Obes Surg 2004;14:318-324.

37. Wax JR, Wolff R, Cobean R, Pinette MG, Blackstone J, Cartin A: Intussusception complicating pregnancy following laparoscopic Roux-en-Y gastric bypass. Obes Surg 2007;17:977-979.

38. Guelinckx I, Devlieger R, Vansant G: Reproductive outcome after bariatric surgery: a critical review. Hum Reprod Update 2009;15:189-201.

39. Ren D, Li M, Duan C, Rui L: Identification of SH2-B as a key regulator of leptin sensitivity, energy balance, and body weight in mice. Cell Metab 2005;2:95-104.

40. Froguel P, Blakemore AI: The power of the extreme in elucidating obesity. N Engl J Med 2008;359:891-893.

41. Ashcroft FM: Adenosine 5'-triphosphate-sensitive potassium channels. Annu Rev Neurosci 1988;11:97-118.

42. Miki T, Minami K, Zhang L, Morita M, Gonoi T, Shiuchi T, et al: ATP-sensitive potassium channels participate in glucose uptake in skeletal muscle and adipose tissue. Am J Physiol 2002;283:E1178-E1184. 
43. Bastie CC, Zong H, Xu J, Busa B, Judex S, Kurland IJ, et al: Integrative metabolic regulation of peripheral tissue fatty acid oxidation by the SRC kinase family member Fyn. Cell Metab 2007;5:371-381.

44. He Z, Tang F, Ermakova S, Li M, Zhao Q, Cho YY, et al: Fyn is a novel target of (-)-epigallocatechin gallate in the inhibition of JB6 Cl41 cell transformation. Mol Carcinog 2008;47:172-183.

45. Thielecke F, Boschmann M: The potential role of green tea catechins in the prevention of the metabolic syndrome - a review. Phytochemistry 2009;70:11-24.

46. Shixian Q, VanCrey B, Shi J, Kakuda Y, Jiang Y: Green tea extract thermogenesis-induced weight loss by epigallocatechin gallate inhibition of catechol-O-methyltransferase. J Med Food 2006;9:451-458.

47. Dulloo AG, Seydoux J, Girardier L, Chantre P, Vandermander J: Green tea and thermogenesis: interactions between catechin-polyphenols, caffeine and sympathetic activity. Int J Obes Relat Metab Disord 2000;24:252-258.

48. Rothwell NJ, Stock MJ: A role for brown adipose tissue in diet-induced thermogenesis. Nature 1979;281:31-35.

49. Enerback S, Jacobsson A, Simpson EM, Guerra C, Yamashita H, Harper ME, et al: Mice lacking mitochondrial uncoupling protein are cold-sensitive but not obese. Nature 1997;387:90-94.

50. Feldmann HM, Golozoubova V, Cannon B, Nedergaard J: UCP1 ablation induces obesity and abolishes diet-induced thermogenesis in mice exempt from thermal stress by living at thermoneutrality. Cell Metab 2009;9:203-209.

51. Atit R, Sgaier SK, Mohamed OA, Taketo MM, Dufort D, Joyner AL, et al: Beta-catenin activation is necessary and sufficient to specify the dorsal dermal fate in the mouse. Dev Biol 2006;296:164-176.

52. Seale P, Bjork B, Yang W, Kajimura S, Chin S, Kuang S, et al: PRDM16 controls a brown fat/skeletal muscle switch. Nature 2008;454:961-967.

53. Seale P, Kajimura S, Yang W, Chin S, Rohas LM, Uldry M, et al: Transcriptional control of brown fat determination by PRDM16. Cell Metab 2007;6:38-54.

54. Ley RE, Peterson DA, Gordon JI: Ecological and evolutionary forces shaping microbial diversity in the human intestine. Cell 2006;124:837-848.

55. Turnbaugh PJ, Ley RE, Hamady M, Fraser-Liggett CM, Knight R, Gordon JI: The human microbiome project. Nature 2007;449:804-810.

56. Slack E, Hapfelmeier S, Stecher B, Velykoredko Y, Stoel M, Lawson MA, et al: Innate and adaptive immunity cooperate flexibly to maintain host-microbiota mutualism. Science 2009;325:617-620. 